\title{
La simulación en la enseñanza en Odontología. Una herramienta de aprendizaje para la seguridad del paciente y la calidad de atención
}

Simulation in education in dentistry. Learning tool for patient safety and

quality of care
Simulação na educação em odontologia. Aprendizagem de ferramentas para a seguran-

ça do paciente ea qualidade dos cuidados

\section{Juan José Christiani}

Doctor en Odontología.

Docente de la asignatura Preclínica de Prótesis.

Facultad de Odontología.

Universidad Nacional del Nordeste.

Email: jjchristiani@odn.unne.edu.ar

Lugar de Trabajo Facultad de Odontología.

Avda Libertad 5450. CP. 3400 Corrientes.

Argentina

\section{Resumen}

La simulación clínica en odontología es una herramienta de aprendizaje cuyo objetivo principal es la adquisición de competencias en un ámbito lo más parecido al contexto real. El uso de simuladores es una herramienta educativa con la que se favorece la adquisición de ciertas habilidades, destrezas, técnicas y competencias necesarias para la formación del estudiante o profesional. La simulación en odontología antes de un ciclo clínico favorece el análisis crítico posterior a la práctica, pudiendo utilizarse para reproducir diferentes casos de rehabilitaciones orales que tendrá que enfrentar el estudiante luego en la clínica y de esta manera poder observar el grado de dominio alcanzado por los alumnos.

La metodología de la simulación permite el mejoramiento continuo en la calidad de la atención de los pacientes, centrado en diferentes aspectos de desempeño de los estudiantes, tales como habilidades, técnicas comunicacionales y actitudinales, preparándolo para enfrentar una atención segura y de calidad.

\section{Palabras claves}

Simulación, herramienta, enseñanza, calidad.

\section{Summary}

Clinical simulation in dentistry is a learning tool whose main objective is the acquisition of skills in as close to real context field. The use of simulators is an educational tool that the ac- 
quisition of certain skills, skills, techniques and skills required for student training or professional favors. The simulation in dentistry before a clinical cycle favors the subsequent critical analysis into practice, can be used to play different cases of oral rehabilitations that will face the student then at the clinic and thus able to observe the degree of dominance reached by students.

The simulation methodology allows for continuous improvement in the quality of patient care, focusing on different aspects of student performance, such as skills, communication techniques and attitude, preparing to face a safe, quality care.

\section{Keywords}

Simulation, tool, teaching quality.

\section{Resumo}

Simulação clínica em odontologia é uma ferramenta de aprendizagem cujo principal objectivo é a aquisição de competências em tão perto de campo de contexto real. A utilização de simuladores é uma ferramenta educacional que a aquisição de competências certas, habilidades técnicas e habilidades necessárias para a formação do estudante ou favores profissionais. A simulação em odontologia antes de um ciclo clínico favorece a análise crítica subsequente em prática, pode ser usado para reproduzir diferentes casos de reabilitações orais que irá enfrentar o aluno, em seguida, na clínica e, portanto, capaz de observar o grau de domínio alcançado pelo alunos.

A metodologia de simulação permite a meIhoria contínua na qualidade do atendimento ao paciente, com foco em diferentes aspectos do desempenho dos alunos, tais como habilidades, técnicas de comunicação e atitude, preparando-se para enfrentar, a cuidados de qualidade segura.

\section{Palavras chave}

Simulação, ferramenta, a qualidade do ensino.

\section{Introduccion}

La simulación clínica es una herramienta de aprendizaje cuyo objetivo principal es la adquisición de competencias en un ámbito lo más parecido al contexto real.

La seguridad del paciente definida como la disminución o ausencia de daño durante el proceso de atención en salud en los últimos años ha sido centro de las políticas sanitarias como un elemento de su calidad y mejora.'

La seguridad del paciente ha aumentado las expectativas sobre la responsabilidad de los docentes en salud para asegurar el logro de las competencias. El objetivo principal de los profesionales de la salud debe ser la prestación de la atención con la mejor calidad posible a los pacientes'. La educación en salud es un medio fundamental para lograr este objetivo ayudando a los estudiantes y profesionales a desarrollar una gama adecuada de habilidades, conocimientos y actitudes.

El aprendizaje mediante simulación se utiliza para el entrenamiento y la formación de los alumnos en las ciencias de la salud así como para la formación de profesionales en nuevos procedimientos y tecnología.

La metodología de simulación clínica permite el mejoramiento continuo en la calidad de la atención de los pacientes, centrado en diferentes aspectos, tales como habilidades, técnicas actitudinales, que permiten fortalecer una atención segura y adecuada para todos los pacientes $^{2}$. Asimismo, la enseñanza objetiva y sistemática de las habilidades psicomotoras y cognitivas en odontología y en las demás profesiones de las ciencias de la salud puede ser realizada con el auxilio de estas herramientas como la simulación, definida como la representación artificial de procesos reales, con la suficiente fidelidad para alcanzar una meta en particular, sea ésta el entrenamiento del individuo o la evaluación de sus habilidades ${ }^{3-7}$.

Los simuladores en odontología favorece no solamente el desarrollo de competencias correspondientes a procesos técnicos de la práctica profesional, sino que, tiene una utilidad que va mucho más allá de lo técnico y tecnológico y lo que es más importante, apoya el desarrollo de competencias profesionales ${ }^{6}$. 
A través de la simulación, centramos el objeto del conocimiento en el caso clínico (paciente con necesidad de rehabilitación oral ) tratando de reproducir ese universo concreto, característico, real, en un universo simbólico que queda en el alumno ya sea como representaciones mentales, símbolos o signos, valores y/o modelos de comportamiento que facilitan en el momento que enfrente la realidad clínica una estructuración física y mental para actuar idóneamente a pesar de no tener la experiencia real previa o con una organización mental ideal en el momento de tener que enfrentar la clínica.

La formación del odontólogo depende del modelo pedagógico asumido que condiciona la incorporación de simulación dentro del proceso aprendizaje, la cual puede ser visualizada en prácticas previas a la inserción del estudiante en actividades clínicas.

El objetivo de la simulación en odontología es contar con una herramienta educativa que favorezca la adquisición de ciertas habilidades, destrezas, técnicas y competencias necesarias para la formación del estudiante o profesional de odontología.

\section{Desarrollo}

La capacitación y entrenamiento en un ciclo preclínico por medios simuladores en odontología conlleva a preparar al profesional o estudiante a encontrarse con situaciones reales en la clínica. A diferencia de los oclusores que se utilizaban para realizar las prácticas preclínicas, en la actualidad existen simuladores con movimientos mandibulares, con dientes artificiales para las prácticas en las diferentes especialidades y en otras aéreas de la odontología preparando a los estudiantes en simuladores clínicos donde las prácticas y el contexto se asemejan más a la realidad.

Mediante la simulación se certifican ciertas habilidades y destrezas que se requieren en la profesión con el fin de promover una cultura de seguridad del paciente ${ }^{7-9,10}$.

La seguridad del paciente en las ciencias de la salud tomo estrategias de la industria de la aviación en donde usan la simulación para el entrenamiento de habilidades y control de situaciones críticas, sin poner en riesgo a las personas.
Existen diversos tipos de simulación en salud que son utilizados como estrategia de aprendizaje, entre estos se encuentran el paciente estandarizado, el simulador humano, los simuladores virtuales y los simuladores de habilidades ${ }^{10,11}$

Los simuladores actuales para odontología se presentan con cabeza móvil con torso superior, cuentan con mejillas, lengua y los maxilares, con sus respectivas piezas dentarias pudiendo abrir y cerrar la boca, así como también simuladores para técnicas anestésicas, para procedimientos quirúrgicos lo que favorece el análisis crítico posterior a la realización de la práctica, pudiendo utilizarse para reproducir diferentes situaciones de rehabilitación oral para posteriormente observar el grado de dominio alcanzado por los alumnos.

Una de las grandes diferencias entre la enseñanza en la salud con el modelo tradicional y la enseñanza basada en la simulación, es que durante el entrenamiento clínico, en pacientes, los alumnos deben de estar continuamente supervisados para evitar que cometan errores y corregirlos de manera inmediata, esto con el fin de cuidar la integridad y seguridad del paciente, dentro de una simulación, los errores si ocurrieron se lo hace observar, con el fin de que el alumno aprenda de las consecuencias de su error, rectifique y vuelva a realizar el procedimiento de manera correcta, reforzando así sus conocimientos.

Una de las características de la enseñanza con simuladores es que en ésta se utiliza el aprendizaje previamente adquirido para estimular la participación del alumno, potenciar el conocimiento cercano a la vida real y su aplicación a situaciones cotidianas. Estos conceptos conducen hacia las cuatro características básicas de la simulación: la observación del mundo real, su representación física o simbólica, la acción sobre esta representación y los efectos de esta acción sobre el aprendizaje humano.

Asimismo para que la simulación clínica sea una herramienta adecuada es necesario se realizan prácticas suficiente y se hagan la evaluación de los resultados. El empleo de la simulación permite acelerar el proceso de aprendizaje y contribuye a elevar su calidad.

El uso de simuladores en odontología es una herramienta de enseñanza para que los estudiantes puedan desarrollar actitudes en pacientes no reales o simuladores que pueden contar con 
\begin{tabular}{r|l} 
REVISTA FACULTAD DE ODONTOLOGÍA & OPINIÓN \\
ISSN No 1668-7280 - Vol. IX No 1-2016 & $\begin{array}{l}\text { Christiani } \\
72\end{array}$
\end{tabular}

tecnología avanzada que permite la construcción de escenarios con un alto grado de realismo, sin embargo, la simulación no tiene como propósito encontrar en el simulador la identidad con el paciente ni reemplazar la práctica con pacientes reales, sino el objetivo es preparar a los estudiantes de manera óptima para ella, a través de la generación de una memoria manual, auditiva, visual y sensorial, mediante un proceso repetitivo, sistematizado y organizado con el objetivo de evitar el error, que puede corregirse con el entrenamiento, mejorando los tiempos de ejecución, la calidad del actuar y la seguridad del paciente.

\section{Conclusión}

La simulación es una herramienta que sirve para favorecer la realización de prácticas clínicas. Esta puede ser utilizada como herramienta educativa para evaluar el desempeño del estudiante de odontología en un ambiente seguro y controlado, el cual asemeja al ambiente clínico habitual.

El objetivo de la educación en salud es el desarrollo de profesionales competentes y dedicados que sean capaces de proporcionar el más alto nivel de atención y seguridad a sus pacientes. En los últimos años, la simulación ha entrado en escena de una forma continua y su uso ha crecido de manera exponencial realizándose grandes avances tecnológicos en la inclusión de esta herramienta en los diferentes programas educativos en las ciencias de la salud.

La metodología de la simulación en odontología permite el mejoramiento continuo en la calidad de atención de los pacientes, centrado en diferentes aspectos de desempeño de los estudiantes (habilidades, técnicas) preparándolo para un ciclo clínico que permiten fortalecer una atención segura y adecuada para los pacientes.

\section{Bibliografía}

I. Christiani JJ, Rocha MT, Valsecia M. Seguridad del Paciente en la práctica odontológica. Acta Odontológica Colombiana. 2015;5 (2): 2I-32 [en línea] [fecha de consulta: 03/03/2016]. Disponible desde: http:// www.revistas.unal.edu.co/ index.php/ actaodontocol
2. Dávila Cervantes A. Simulación en la educación médica, Inv Ed Med 2014; 3 (10): I00-I05

3. Salas RS, Ardaza P. La simulación como método de enseñanza y aprendizaje. Rev Cubana Edu Med Sup. 1995; 9(I-2): Disponible en: http//bvs. sld.cu/revistas/ems/vol9_I_95 lems03195.htm. Consultado (II de abril 20I6).

4. Gorman PJ, Meier AH, Raw Ch, Krummel TM. The future of medical education is no longer blood and guts. Am J Sur. 2000 Nov; 180 (5): 353-356.

5. Doyle DJ Simulation in medical education: focus in anesthesiology. Med Edu. 2002. Disponible en: http://www.med-ed-online.org/f0000053.htm. (Consulta: 2016, Abril I6).

6. Buchanan JA, Williams JN. Simulation in dentistry and oral surgery. Loyd GE, Lake CL, Greenberg RB. Practical health care simulations. USA, Elsevier Mosby. 2004. 628 p.

7. Nara N, Beppu M, Tohda S, Suzuki T, The introduction and effectiveness of simulation- based learning in medical education. Int Med. 2009;48: I5I5-1519.

8. Fox-Robichaud AE, Nimmo GR. Education and simulation techniques for improving reliability of care. Curr Opin Crit Care 2007; 13:737-74I.

9. Bond $W$, Khun $G$, Binstadt $E$, et al. The use of simulation in the development of Individual Cognitive Expertise in Emergency Medicine. Acad Emer Med 2008; 15: 1037-1045.

10. Kneebone R. Simulation in surgical training educational issues and practical implications. Med Educ 2003;37: I: 22-28.

II. Ziv A. A Practical Guide for Medical Teachers. Simulators and simulation-based medical education. Elsevier Academic Press; 2009;3:28: 217-222.

12. Barsuk JH, Cohen ER, Feinglass J, et al. Use of simulation-based education to reduce catheterrelated bloodstream infections. Arch Intern Med 2009; 169 (I5): I420-1423.

13. Barsuk JH, Cohen ER, McGaghie WC, et al. Long-term retention of central venous catheter insertion skills after simulation-based mastery learning. Acad Med 2010; 85 (I0 Suppl.): S9-SI2.

14. Motola I, Devine LA, Chung HS, et al. Simulation in health care education: a best evidence practical guide. AMEE Guide No. 82. Med Teach 2013; 35 (I0): el5II-I530. 